\title{
Preoperative Embolization to Improve Surgical Management and Outcome of Pulmonary Sequestration
}

\author{
Vivek Ukirde ${ }^{1}$ Saurabh Joshi ${ }^{1} \quad$ Ashank Bansal $^{1} \quad$ Arvind Borde $^{1} \quad$ Amol Lahoti $^{1}$
}

${ }^{1}$ Department of vascular and interventional radiology, LTM General Hospital and Medical College, Sion, Mumbai, India

\begin{abstract}
Address for correspondence Amol Lahoti, Fellow vascular and interventional radiology (FVNIR), Department of vascular and interventional radiology, LTM General Hospital and Medical College, 502, Shiv Koliwada, Sion Circle, Sion 400022, Mumbai, India (e-mail: amollahoti20031987@gmail.com; amollahoti_203@yahoo.co.in).
\end{abstract}
Abstract
Keywords
- pulmonary sequestration
- vascular plug
- preoperative
- embolization

Pulmonary sequestration is a rare embryonic malformation of lung tissue with no identifiable bronchial communication receiving its blood supply from one or more anomalous systemic arteries or aorta. The authors present a case report of an adult woman who presented with untreatable hemoptysis. She was treated with anti-Koch's therapy for her nonresolving hemoptysis. She underwent preoperative vascular embolization and operative surgical resection.

\section{Introduction}

The term "sequestration" was coined by Pryce ${ }^{1}$ in 1946 as disconnected bronchopulmonary mass or cyst with an anomalous systemic arterial feeder.

Pulmonary sequestration is defined as a segment of lung parenchyma separated from the tracheobronchial tree, receiving blood supply from a systemic artery ${ }^{2}$ (aorta) rather than a pulmonary arterial branch. The arterial supply is usually from the aorta. Two forms are described in the literature ${ }^{1}$ : intralobar sequestration (ILS), in which sequestered part of the lung lies within normal pulmonary visceral pleura and is surrounded by normal lung $^{2}$ and extralobar sequestration (ELS), in which the abnormal segment of the lung is separate and enclosed in its own pleural investment, which means ELS has a separate pleural covering.

\section{Case Report}

A 25-year-old female patient reported to our department with chief complaints of hemoptysis immediately followed by menstruation (though not significant, this was the real complaint with which the patient presented). The patient has history of long-standing untreatable hemoptysis and intermittent chest pain on inspiration. Hemoptysis for 5 years, each episode around $30 \mathrm{~mL}$ fresh blood, has been associated with coughing and $\mathrm{Hb} 6 \mathrm{~g}$. The patient received 6 months of anti-Koch's treatment in view of persistent hemoptysis despite strong and broad-spectrum antibiotics 1 year back. She had pallor and tachycardia. A high-resolution computed tomographic (CT) angiography of the thorax and upper abdomen was done to rule out cause of persistent hemoptysis, which showed a well-defined soft tissue density area in the left lower lobe that is supplied by an aberrant artery originating directly from the abdominal aorta of approximately $6 \mathrm{~mm}$ diameter. The area showed venous drainage in the normal pulmonary veins and showed communication with the bronchi ( - Fig. 1 a, b).

After interdisciplinary meeting, preoperative embolization for aberrant arterial feeder was suggested to reduce intraoperative bleeding and associated risk. On digital subtraction angiography (DSA), an arterial feeder ( - Fig. 2 ) was noted arising directly from the abdominal aorta. The choice of embolic agent was vascular (type 2) plug (-Fig. 3). When we were trying to deploy the vascular plug, the initial position of the plug was just obliterating the upper part of aberrant feeder ( - Fig. 4). After some manipulation and withdrawing the plug, the blood supply was completely stopped
DOI https://doi.org/

$10.1055 / \mathrm{s}-0038-1676670$

ISSN 2455-7420.
License terms

(1) $\Theta \circledast$ 


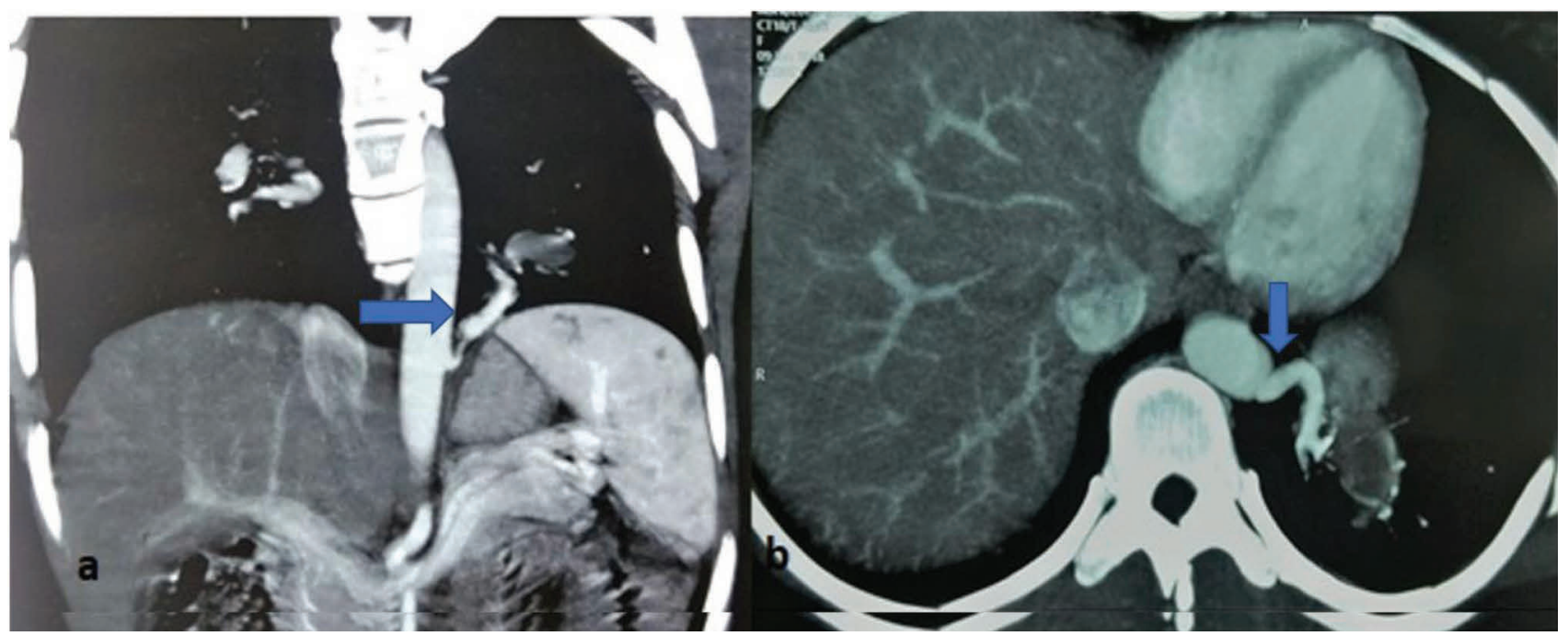

Fig. 1 (a, b) Coronal and axial sections of CT angiography, respectively, showing aberrant feeder (blue arrow) directly arising from aorta and supplying the left lower lobe.

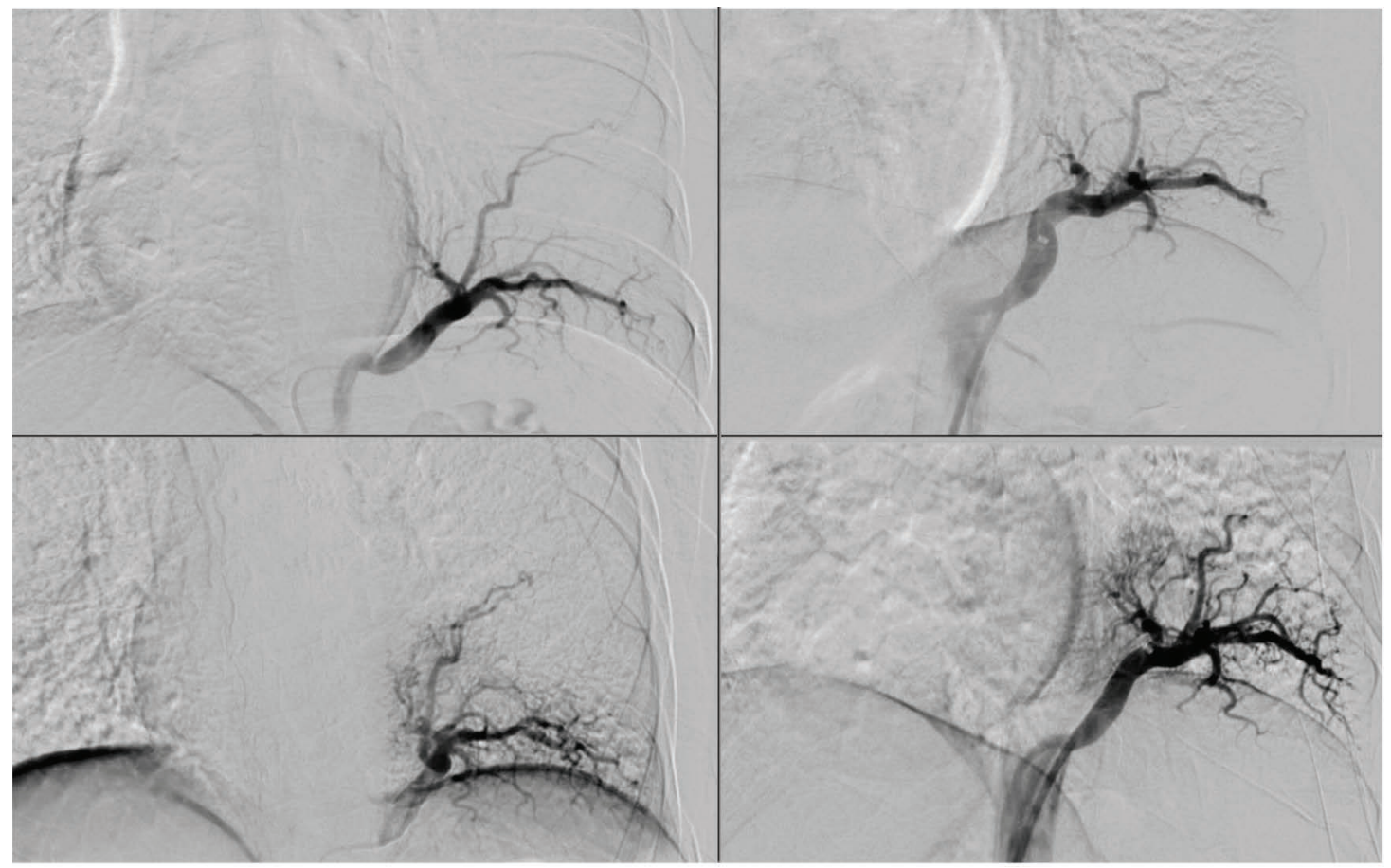

Fig. 2 DSA angiography image showing arterial feeder arising from aorta supplying the sequestration in the left lower lobe.

as observed on DSA scan (-Fig. 5). Intraoperative image ( - Fig. 6) showed the easily held vascular pedicle and sequestered part of the lung. On follow-up, surgical team said, "not only blood loss was significantly decreased but they also got clear field to operate." At 2 months follow-up, our patient remained hemoptysis and pain free.

\section{Discussion}

A bronchopulmonary sequestration (BPS) consists of a section of nonfunctional lung tissue, which is not communicating with the normal bronchial tree and vasculature, and is vascularized by an aberrant systemic artery and venous drainage through the pulmonary veins, azygos system, or inferior vena cava. ${ }^{3}$ Its common site is the lower lobe, more often left than right. Normally no communication with the bronchial tree exists, and anomalous connection with other bronchi or lung parenchyma regularly occurs. However, when this is present, recurrent infections are often seen. ${ }^{4}$

ELSs are located inside their own visceral pleura and mostly found between the lower lobe and diaphragm, more on left side or in the upper abdomen. There is complete separation with other lung tissue, and therefore infections are uncommon. 


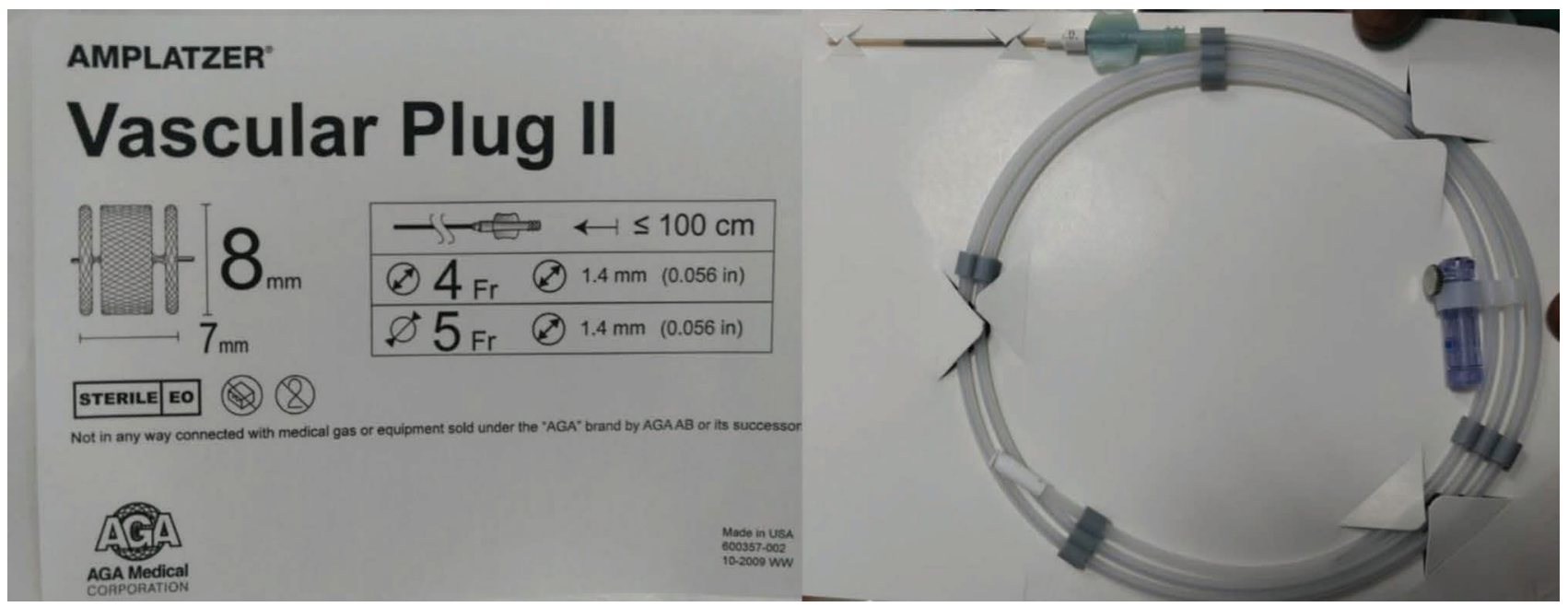

Fig. 3 The $8 \times 7 \mathrm{~mm}$ type II Amplatzer vascular plug used in this case.

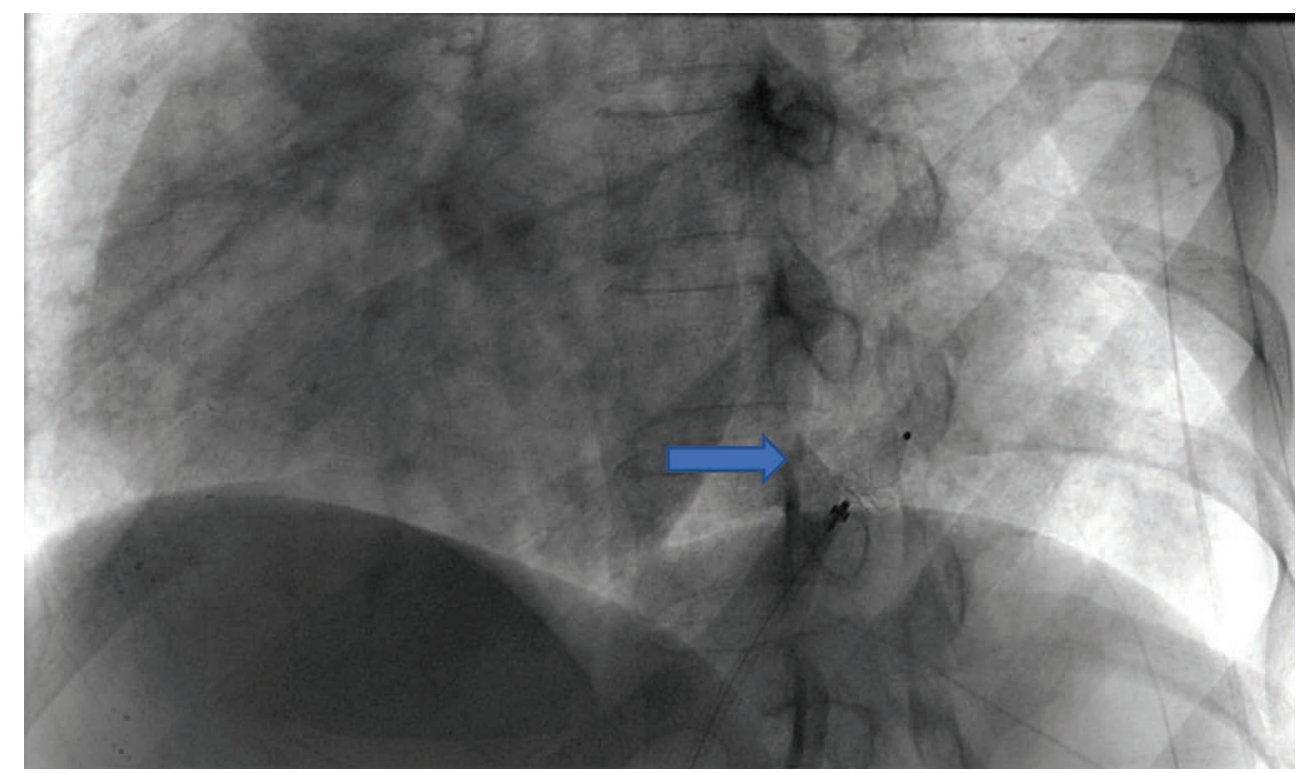

Fig. 4 DSA image showing the vascular plug in situ in the aberrant feeder (blue arrow) that is supplying the sequestration.

ELS patients commonly present with early signs of respiratory distress during childhood, whereas ILS patients are more commonly diagnosed during adolescence or adulthood and show recurrent pneumonias and or hemoptysis. ${ }^{5}$ The diagnosis can be made on a chest radiograph, which shows a dense mass or an air-fluid level. However, often it can be missed when very subtle. However, the investigation of choice is $\mathrm{CT}$ angiography that shows the aberrant artery and venous drainage. ${ }^{6,7}$ The gold standard for treatment of BPS is a surgical ligation and resection. ${ }^{3}$ In ELS, a resection of the sequester is performed by means of video-assisted thoracoscopic surgery (VATS) or a conventional thoracotomy. Embolization as a treatment of arterial bleedings has rapidly become the treatment of the choice for a wide range of indications.
However, for preoperative embolization, there are few options to use such as coils, vascular plug, and coils with vascular plug.

This approach to successfully treat BPS has been reported since $1998 .{ }^{8}$ Selective embolization of the aberrant artery was successfully performed. On follow up, there was significantly reduced perioperative blood loss and clear surgical field.

Clinical significance: This experience emphasizes our conviction that though surgical resection is the treatment of choice in bronchopulmonary sequestration, preoperative embolization helps reduce blood loss and complications associated with excess blood loss.

It is the only treatment in which the abnormal lung tissue can be removed safely giving no opportunity for complications such as ischemic infarction or abscess formation. 


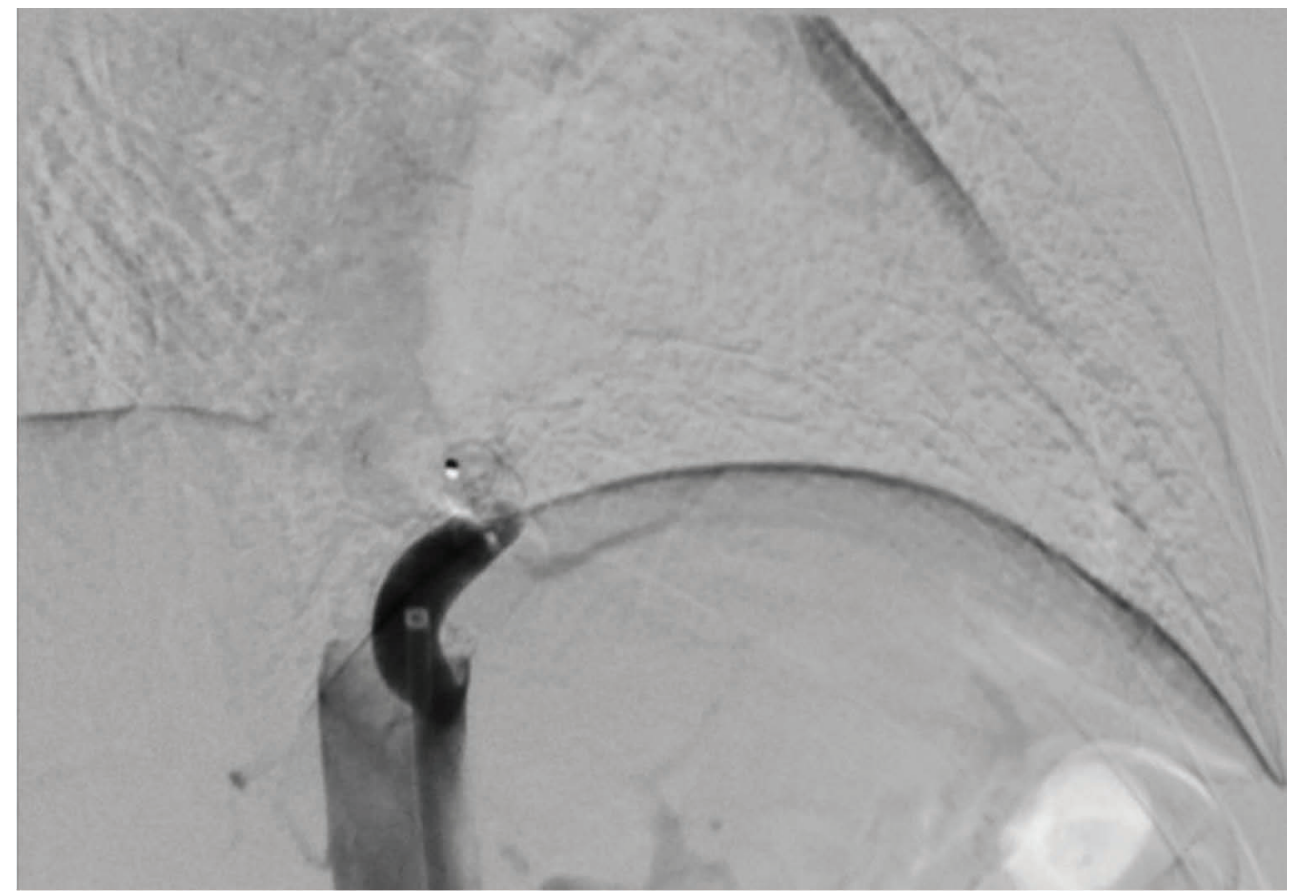

Fig. 5 DSA image showing no blood flow to the sequestered lung suggesting preoperative embolization success.

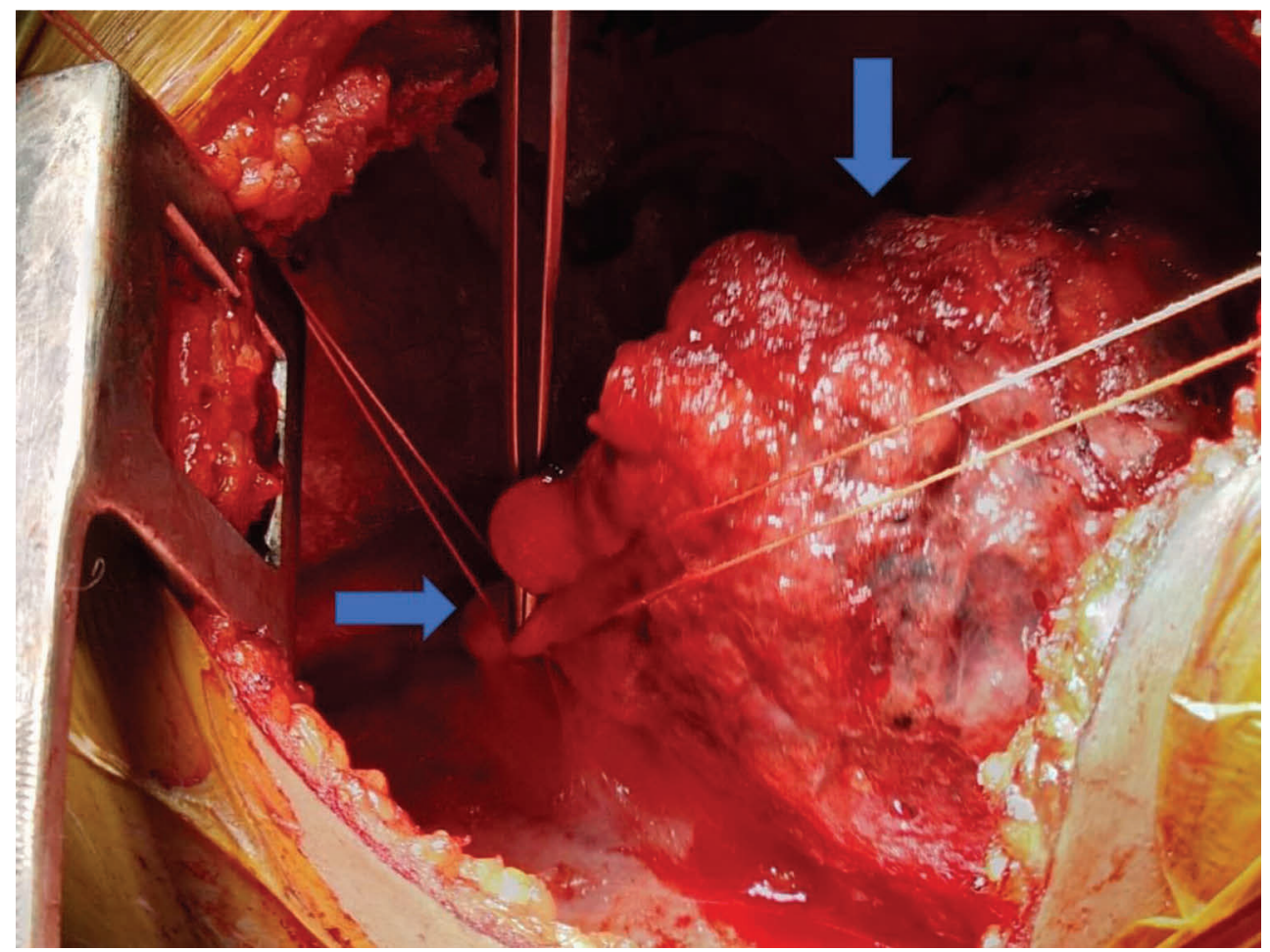

Fig. 6 Intraoperative photo showing the sequestered lung (blue arrow) and its vascular pedicle held (blue arrow). 


\section{Funding}

None.

\section{Conflict of Interest}

None.

\section{References}

1 Felker RE, Tonkin IL. Imaging of pulmonary sequestration. AJR Am J Roentgenol 1990;154(2):241-249 Review

2 DiksJ,SchüttePR,CheungD,SchnaterJM.Treatmentof pulmonary sequestrations by means of endovascular embolization: future or fashion? Case Rep Med 2011;2011:173918

3 Clements BS, Warner JO, Shinebourne EA. Congenital bronchopulmonary vascular malformations: clinical application of a simple anatomical approach in 25 cases. Thorax 1987;42(6):409-416
4 Gezer S, Taştepe I, Sirmali M, et al. Pulmonary sequestration: a single-institutional series composed of 27 cases. J Thorac Cardiovasc Surg 2007;133(4):955-959

5 Miller EJ, Singh SP, Cerfolio RJ, Schmidt F, Eltoum IE. Pryce's type I pulmonary intralobar sequestration presenting with massive hemoptysis. Ann Diagn Pathol 2001;5(2):91-95

6 Kang M, Khandelwal N, Ojili V, Rao KL, Rana SS. Multidetector CT angiography in pulmonary sequestration. J Comput Assist Tomogr 2006;30(6):926-932

7 Hartmann IJ, Remy-Jardin M, Menchini L, Teisseire A, Khalil C, Remy J. Ectopic origin of bronchial arteries: assessment with multidetector helical CT angiography. Eur Radiol 2007;17(8):1943-1953

8 Park ST, Yoon $\mathrm{CH}$, Sung KB, et al. Pulmonary sequestration in a newborn infant: treatment with arterial embolization. J Vasc Interv Radiol 1998;9(4):648-650 